\title{
Assessment of the Relationship Between Red Cell Distribution Width and Brucellosis: A Case- Controlled Study
}

\section{Li Gou}

People's Hospital of Xinjiang Uygur Autonomous Region

\section{Feng Gao}

People's Hospital of Xinjiang Uygur Autonomous Region

\section{Siqin Lan}

Xinjiang Medical University Affiliated First Hospital

Hui Guo ( $\nabla$ guohui9804@126.com )

Xinjiang Medical University Affiliated First Hospital https://orcid.org/0000-0001-8442-6487

\section{Research}

Keywords: Red cell distribution width, Brucellosis, Biochemical markers, Sensitivity, Specificity

Posted Date: May 3rd, 2021

DOI: https://doi.org/10.21203/rs.3.rs-383303/v1

License: (c) (i) This work is licensed under a Creative Commons Attribution 4.0 International License. Read Full License 


\section{Abstract}

Background: As a multisystem infectious disease, there is inflammation, which causes an increase in brucellosis. The red cell distribution width (RDW) has been identified as markers of inflammation. The present study aimed to investigate the predictive contribution value of RDW in the diagnosis of brucellosis.

Methods: Medical records of 398 patients with brucellosis and 398 age-matched and gender-matched healthy controls in a single center from January 2017 to December 2019 were retrospectively reviewed.

Results: The mean age of brucellosis patients was $47.1 \pm 12.9$ years. The RDW levels were significantly higher in brucellosis group when compared to the control group ( $<0.001)$; The RDW levels were markedly lower in brucellosis male patients $14.32 \pm 2.15 \%$ when compared to the brucellosis female patient $s 15.41 \pm 3.13 \%(t=-9.56, p<0.001)$. The biochemical markers of brucellosis patients included erythrocyte, hemoglobin, $\mathrm{HCT}$, corpuscular volume, hemoglobin, hemoglobin concentration were all negatively correlated with RDW $(r=-0.227,-0.383,-0.266,-0.253,-0.311$ and -0.225 , all $p<0.001)$. The values of hemoglobin ( $\beta$ coefficient $=-0.084, p<0.001$ ), ESR ( $\beta$ coefficient $=-0.020, p=0.004$ ) were significantly and independently correlated with RDW. Receiver operating characteristic (ROC) curve analysis showed that the best cutoff point for RDW in the diagnosis of brucellosis was $13.45 \%$, which evaluated brucellosis with a sensitivity of $64.1 \%$ and a specificity of $83.9 \%$. The area under the ROC curve for RDW was calculated as $0.80(95 \% \mathrm{Cl} 0.769-0.831, \mathrm{P}<0.001)$.

Conclusion: This study revealed that the RDW in diagnosing brucellosis had a higher sensitivity and specificity. RDW values may be useful complementary indirect markers for the diagnosis of brucellosis.

\section{Introduction}

Brucellosis is a common zoonotic infection worldwide, affecting more than 500000 people each year. Its prevalence is more than 10/100 000 population in some endemic regions [1]. In China, brucellosis is common; the incidence of human brucellosis is estimated to vary from $<0.03$ to $>160$ individuals per 100000 population [2]. Although its true incidence in China remains largely unknown, it is thought that this increase results from improvements in diagnosis and increased reporting.

Brucellosis has been prevalent for several years in China, and human brucellosis was distributed in 25 of 32 provinces or autonomous regions of China, especially in Xinjiang region. Consequently, this disease is a high economic and public health problem for sufferers [3].

Clinical diagnosis can be challenging, particularly in the early stages of brucellosis, when clinical manifestations may be quite atypical or non-specific. Different elements of history, laboratory, and examination findings have varied predictive power in diagnosing brucellosis, and clinical evaluation exists, but brucellosis can be easily overlooked. The RDW is an objective measurement, which reveals the variability of circulating red blood cells. In the past several years, RDW has received the attention in the 
field of inflammation in as much as it was associated with unfavorable clinical outcomes in patients with myocardial infarction, acute pancreatitis-associated lung injury, polycystic ovary syndrome, spontaneous echo contrast, and ankylosis spondylitis [4-8], however, few studies about brucellosis in literature have examined this subject before. According to the literature, it is not exactly clear what changes occur in RDW in brucellosis. In the present study, we aimed to seek whether RDW levels are related to diagnosis brucellosis.

\section{Materials And Method}

This is a case-controlled retrospective clinical study. Data were collected from patients' records archived in Xinjiang medical university affiliated the first hospital from January 2017 to December 2019. In this time frame, there was a record of 398 patients. We then included 398 age-matched and gender-matched healthy individuals. This study was performed in accordance with the principles of the declaration of Helsinki. It was reviewed and approved by an institutional review board exemption and a waiver for the requirement of the written informed consent. We collected patients' blood samples and clinical symptoms at the admission, and then we analyzed laboratory parameters. The diagnoses depended on the combination of medical history, brucella agglutination titer test, and the exclusion of other inflammatory diagnoses.

\section{Laboratory Measurements}

Hemogram parameters including white blood cell (WBC), neutrophil, lymphocyte, mononuclear, red blood cell (RBC), hemoglobin (HGB), hematocrit (HCT), mean corpuscular volume (MCV), mean corpuscular hemoglobin (MCH), mean corpuscular hemoglobin concentration (MCHC), RDW, C-reactive protein (CRP), erythrocyte sedimentation rate (ESR), agglutination assay and culture for blood admission were also recorded. Neutrophil to lymphocyte ratio (NLR) was calculated as the ratio of neutrophils to lymphocytes. These hematological variables were measured and recorded in healthy individuals as well.

\section{Inclusion Criteria}

Brucellosis patients were based on the brucella serum agglutination test $(\geq 1: 160)$. All patients and healthy individuals' were no less than 18 years old. There were no other acute and chronic infections. Patients were matched $1: 1$ by age and gender with healthy individuals who had the medical examination in our center.

\section{Exclusion Criteria}

Patients were excluded from the study if they were confirmed for severe cardiovascular disease, significant liver disease, neoplasms, autoimmune disorders, other chronic inflammatory conditions, and being immunocompromised. Patients were excluded from the study if they had been treated before we collected patients' blood samples. Further exclusion criteria were if the patient's blood data were not complete. Patients cannot be confirmed by any means, and had a history of surgery were excluded. 


\section{Statistical analysis}

Statistical analysis was performed with SPSS17.00 statistical software. Continuous variables were shown as mean \pm standard deviation, and categorical variables were presented as a percentage. The groups were compared using the t-test for continuous variables and chi-square test for categorical variables. Correlations between 2 continuous variables were evaluated with the Pearson test. A simple correlation test (Spearman's test) was used to observe the correlation between two variables. A multivariate linear regression analysis with stepwise selection of covariance was used to explore the correlation between RDW and other variables. All candidate variables were checked to see if their significance has been reduced below the specific level. If a non-significant variable was found, it is removed from the analysis. In addition, receiver operating characteristics (ROC) curve analysis was used to measure the performance of RDW. A p-value of less than .05 was considered statistically significant.

\section{Results}

\section{Demographic and clinical characteristics in patients with Brucellosis}

A total of 398 patients were included in brucellosis group, and 398 healthy individuals were included in the control group, making up a total of 796 subjects. Demographic and clinical characteristics of the patient group are shown in Table 1. The mean age of brucellosis patients was 47 years, and ages ranged from 18 to 82 years. The ratio of males to females is 3 to 1 . The most common brucellosis symptoms at the time of diagnosis were fever (61.6\%), followed by fatigue (48.2\%), anorexia (43.7\%), sweating (40.7\%), weight loss (39.2\%), arthralgia (35.4\%), nausea and vomiting (30.4\%), weakness $(29.4 \%)$, headache (24.1\%), abdominal pain (20.4\%), chest pain (13.3\%), myalgia (10.8\%), back pain $(9.3 \%)$, lumbago (7.8\%), diarrhea (6.3\%), abortion (5.3\%), and neck pain(3.5\%), respectively (Table 1). 
Table 1

Demographic and clinical characteristics in patients with Brucellosis

Clinical features Mean \pm SD, median range or number $(\%)(n=398)$

Mean age (year) $47.1 \pm 12.9$

Gender

Male $296(74.4)$

Female 102 (25.6)

Mean symptom duration (day) 8(2-106)

Clinical symptoms

Fever 245(61.6)

Fatigue 192 (48.2)

Anorexia 174 (43.7)

Sweating 162 (40.7)

Weight loss 156 (39.2)

Arthralgia 141 (35.4)

Nausea and vomiting 121 (30.4)

Weakness 117 (29.4)

Headache 96 (24.1)

Abdominal pain 81 (20.4)

Chest pain 53 (13.3)

Myalgia 43(10.8)

Back pain 37 (9.3)

Lumbago 31 (7.8)

Diarrhea 25 (6.3)

Abortion 21(5.3)

Neck pain 14 (3.5)

Restriction of movement 26 (6.5)

Complications

Sacroiliitis 16 (4.0)

Arthritis 46 (11.6)

Note: SD = Standart deviation 


\section{Clinical features Mean \pm SD, median range or number $(\%)(n=398)$}

Epididymo-orchitis 22 (5.5)

Hepatomegaly $82(20.6)$

Splenomegaly 73 (18.3)

Note: $\mathrm{SD}=$ Standart deviation

\section{Laboratory Findings Of Brucellosis Patients And Healthy Individuals}

Laboratory findings of brucellosis patients and healthy individuals are shown in Table 2. The lymphocyte, RBC, RDW, ESR, and CRP values were significantly higher in brucellosis group when compared to control group ( $p<0.001, p=0.005, p<0.001, p<0.001, p<0.001$, respectively); The neutrophil, HGB, MCV, HCT, $\mathrm{MCH}, \mathrm{MCHC}, \mathrm{NLR}$ values were significantly lower in brucellosis group when compared to control group ( $\mathrm{p}$ $=0.003, p=0.023, p=0.015, p<0.001, p<0.001, p=0.002, p<0.001$, respectively); The WBC, mononuclear cell values was not significantly different between two groups $(P>0.05)$. The results demonstrated that male patients with brucellosis had significantly lower values of RDW $(14.32 \pm 2.15 \%$ vs. $15.41 \pm 3.13 \%, t=-9.56, p<0.001)$ than female patients with brucellosis. 
Table 2

Laboratory findings of the brucellosis patients and healthy individuals

\section{Parameters Study group mean \pm SD Control group mean \pm SD $p$-value or median range or median range}

WBC $(\mathrm{K} / \mu \mathrm{L}) 6.44 \pm 3.139 .09 \pm 3.030 .154$

Neutrophil $(103 / \mu \mathrm{L}) 3.93 \pm 3.356 .74 \pm 2.990 .003$

Lymphocyte $(103 / \mu \mathrm{L}) 1.99 \pm 0.981 .58 \pm 0.62<0.001$

Mononuclear cell $0.53 \pm 0.280 .65 \pm 0.280 .953$

RBC $4.36 \pm 0.684 .27 \pm 0.780 .005$

HGB, g/dL $124.46 \pm 19.88127 .88 \pm 22.590 .023$

HCT $3.12 \pm 9.7912 .87 \pm 18.50<0.001$

MCV $86.75 \pm 6.5590 .93 \pm 5.670 .015$

$\mathrm{MCH} 28.59 \pm 2.5430 .14 \pm 2.17<0.001$

MCHC $329.36 \pm 14.09331 .33 \pm 12.430 .002$

$\mathrm{RDW}, \% 14.69 \pm 2.8312 .93 \pm 1.34<0.001$

NLR $2.39 \pm 2.545 .39 \pm 4.12<0.001$

CRP $(\mathrm{mg} / \mathrm{L}) 18.70 \pm 28.75(323) 7.73 \pm 15.72(277)<0.001$

$\operatorname{ESR}(\mathrm{mm} / \mathrm{hr}) 38.37 \pm 20.56(347) 11.66 \pm 5.16(278)<0.001$

Note: $\mathrm{WBC}=$ White blood cell, $\mathrm{RBC}=$ Red blood cell, $\mathrm{HGB}=$ Hemoglobin, $\mathrm{HCT}=$ Hematocrit, $\mathrm{MCV}=$ Mean corpuscular volume, $\mathrm{MCH}=$ Mean corpuscular hemoglobin, $\mathrm{MCHC}=$ Mean corpuscular hemoglobin concentration, RDW $=$ Red blood cell distribution width, NLR = Neutrophil to lymphocyte ratio, SD = Standart deviation

\section{Correlations Between RDW And Biochemical Markers In Brucellosis Patients}

The biochemical markers of brucellosis patients included RBC, HGB, HCT, MCV, MCH, MCHC were all negatively correlated with RDW $(r=-0.227,-0.383,-0.266,-0.253,-0.311$ and -0.225 , all $p<0.001)$ (Table 3).

Table 3 Correlations between RDW and biochemical markers in patients with brucellosis 


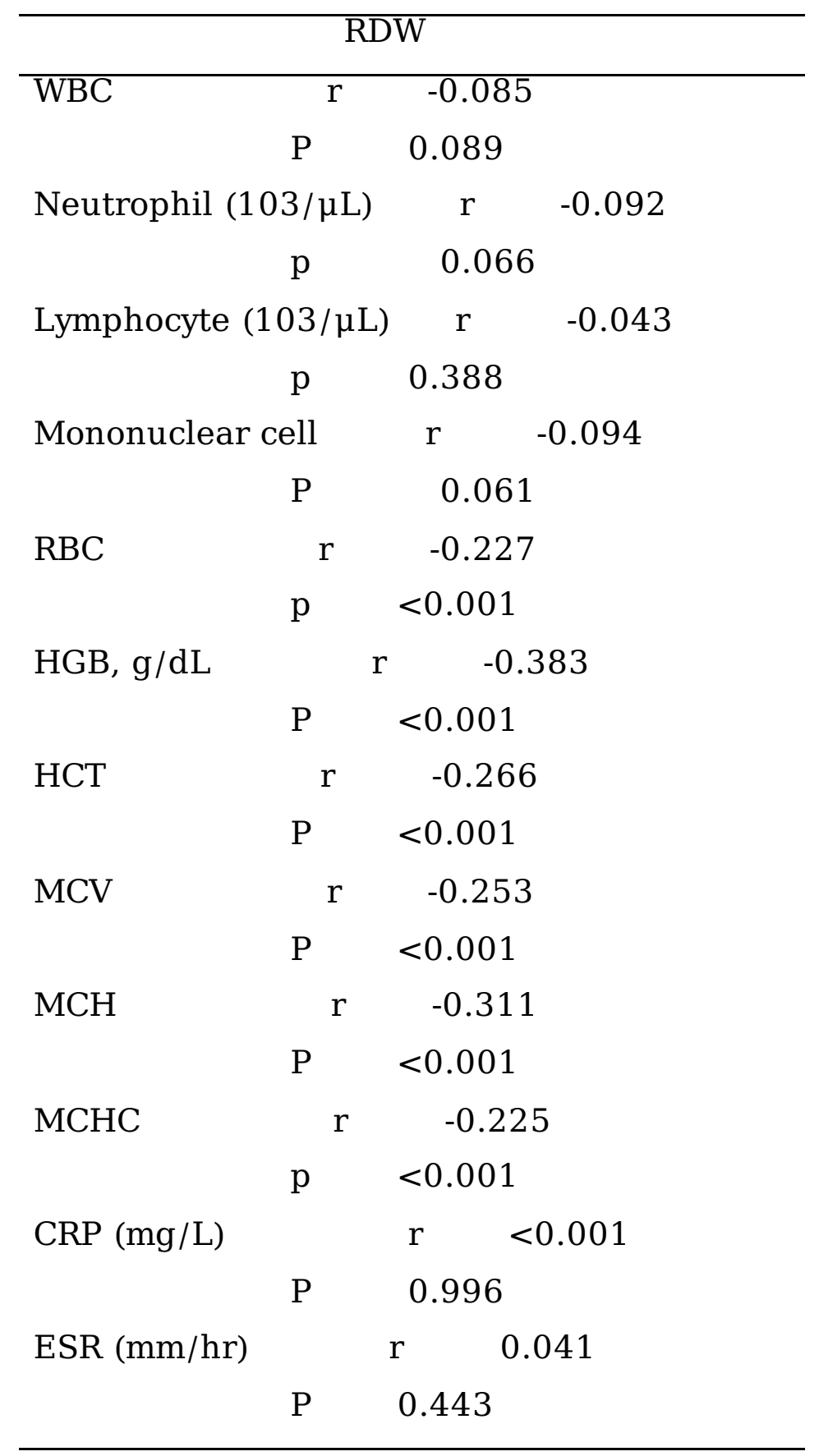

Note: $\mathrm{WBC}=$ White blood cell, $\mathrm{RBC}=$ Red blood cell, $\mathrm{HGB}=$ Hemoglobin, $\mathrm{HCT}=$ Hematocrit, $\mathrm{MCV}=$ Mean corpuscular volume, $\mathrm{MCH}=$ Mean corpuscular hemoglobin, $\mathrm{MCHC}=$ Mean corpuscular hemoglobin concentration, RDW=Red blood cell distribution width, NLR= Neutrophil to lymphocyte ratio

\section{Multivariate Linear Regression Analysis}

According to a multivariate linear regression analysis, values of HGB ( $\beta$ coefficient $=-0.084, p<0.001)$, ESR ( $\beta$ coefficient $=-0.020, p=0.004$ ) were significantly and independently correlated with RDW (Table 4). 
Table 4

A multivariate linear regression analysis of RDW

\begin{tabular}{|l|}
\hline$\beta$ P value \\
Age 0.0030 .812 \\
WBC 0.0770 .742 \\
Neutrophil $(103 / \mu \mathrm{L})-0.1260 .607$ \\
Lymphocyte $(103 / \mu \mathrm{L}) 0.0890 .656$ \\
Mononuclear cell 0.3880 .617 \\
RBC 0.7190 .234 \\
HGB, g/dL -0.084 <0.001 \\
HCT 0.0140 .306 \\
MCV 0.1340 .672 \\
MCH -0.438 0.658 \\
MCHC 0.0340 .688 \\
CRP (mg/L) 0.0040 .367 \\
ESR (mm/hr) -0.020 0.004 \\
NLR -0.009 0.905 \\
\hline Note: WBC = White blood cell, RBC= Red blood cell, HGB = Hemoglobin, \\
\hline HCT = Hematocrit, MCV = Mean corpuscular volume, MCH = Mean \\
\hline corpuscular hemoglobin, MCHC= Mean corpuscular hemoglobin \\
\hline concentration, RDW = Red blood cell distribution width, NLR= \\
\hline Neutrophil to lymphocyte ratio \\
\hline
\end{tabular}

\section{Receiver Operating Characteristic Curve Analysis}

Receiver operating characteristic curve analysis showed that the best cutoff point for RDW in the diagnosis of brucellosis was $13.45 \%$, which evaluated brucellosis with a sensitivity of $64.1 \%$ and a specificity of $83.9 \%$. The area under the ROC curve for RDW was calculated as $0.80(95 \% \mathrm{Cl} 0.769-0.831$, $\mathrm{P}<0.001$ ) (Fig. 1).

\section{Discussion}


The present study revealed that they were significantly higher in the RDW, ESR, CRP and significantly lower in NLR of brucellosis patients when compared to healthy individuals. Moreover, a bivariate and multivariate linear regression analysis demonstrated that RDW was correlated significantly with biochemical markers, indicating that the RDW was heavily associated with brucellosis patients. The ROC curve analysis showed that the RDW in the diagnosis of brucellosis has a higher sensitivity and specificity.

The onset of clinical brucellosis can be acute or insidious. Presenting complaints are often nonspecific symptoms such as fever, nocturnal malodorous diaphoresis, arthralgia, headache, malaise, anorexia, and myalgia [9]. The untreated fever pattern may be intermittent or remittent. Hepatosplenomegaly and lymphadenopathy may be found as well as signs and symptoms associated with other infected organs. In this study, the most common symptoms were fever, followed by fatigue, anorexia, sweating, weight loss, arthralgia, nausea and vomiting, weakness, headache, abdominal pain, chest pain, myalgia, back pain, lumbago, diarrhea, abortion, and neck pain, respectively. The study by Galinska [9] et al. was similar to ours, and the survey by Olt [10] et al. was inconsistent with ours. The reason may be related to their small sample size.

A parameter with the ability to establish the diagnosis of brucellosis has always been a center of attention for physicians. Many different parameters have been examined or are under active investigation for that purpose [11-15]. As for RDW, it is a measure of heterogeneity in the size of circulating red blood cells. It is one of the standard complete blood count components. It has been previously observed that RDW levels have clinical outcomes in various pathologies such as coronary artery disease, pulmonary hypertension, diabetes mellitus, stroke, heart failure, pulmonary embolism, acute pancreatitis, bacteremia, rheumatoid arthritis, inflammatory bowel disease, colon cancer, and celiac disease [16-21].

Although there are a few studies about the hematologic effects of the brucellosis in literature [15, 22, 23], not precisely clear pathogenesis of alteration in RDW, which may be associated with morbidity in this disease, is still lacking. Our findings showed that RDW levels were significantly higher in brucellosis group when compared to the control group. A study by Patel et al. [24] was similar to our results, and it was significantly higher in RDW and significantly lower in NLR levels of brucella epididymo-orchitis when compared to non-brucella epididymo-orchitis. The study by Tekin et al. [22] and the study by Küçükbayrak et al. [16] was similar to ours as well. However, Togan et al.

[15] reported RDW level was not significantly higher in the acute brucellosis patients compared to that of the controls. This finding may be the result of greater RDW levels in chronic inflammatory diseases compared to that in acute conditions. Although the exact pathophysiological basis of the relationship is unclear, chronic inflammation, are proposed underlying factors in this topic [24]. In addition, our survey found that RDW levels were significantly higher in female patients when compared to male patients.

Some studies found that elevations in RDW levels are associated with impaired erythropoiesis or erythrocyte degradation $[18,19]$. In the current study, RDW levels were negatively correlated with RBC, $\mathrm{HGB}, \mathrm{HCT}, \mathrm{MCV}, \mathrm{MCH}, \mathrm{MCHC}$ of the biochemical markers with brucellosis patients. Lack of RBC 
subgroups occurs in parallel with the increase in RDW. Moreover, the neutrophil count decreases when lymphocyte count increases. RDW increases in inflammatory conditions, and this increase are considered as an indicator of systemic inflammation.

Elevated RDW are be related to increased inflammatory markers, such as ESR and CRP, and a strong correlation of RDW with inflammatory markers, ESR, and CRP value has also been found [25-27]. In a study by Lippi et al. [27], a graded association of RDW with hs-CRP and ESR was reported, independent of various confounding factors. In the current study, RDW levels were significantly and independently correlated with HGB, ESR values.

Few studies had analyzed RDW with inflammatory markers of disease by the ROC curve analysis $[18,23]$. In our current study, ROC curve analysis, RDW had an area under the curve of $>0.8$, and the best cutoff point for RDW in the diagnosis of brucellosis was $13.45 \%$, which evaluated brucellosis with a sensitivity of $64.1 \%$ and a specificity of $83.9 \%$. A study by Patel et al. [24] was not similar to our results. The area under a curve, specificity was higher in the present study when compared to a study by Patel et al. The reason may be our large sample patients and the different populations.

\section{Limitations}

The major limit of the current study was the retrospective study that was considered. Single blood sampling was the other limitation of the course. For these reasons, new prospectively controlled and randomized trials with multiple blood, sampling must be performed to confirm our results. Despite that we have suggested that high RDW may be useful data for brucellosis patients.

\section{Conclusion}

The present study showed that RDW levels were higher in patients with brucellosis, and RDW in the diagnosis of brucellosis had a higher sensitivity and specificity. RDW can be useful complementary indirect markers for the diagnosis of brucellosis. There is still a need for further prospective, multicenter studies with a large sample size to fully clarify the issue.

\section{Abbreviations}

RDW Red cell distribution width

HCT Hematocrit

ESR Erythrocyte sedimentation rate

ROC Receiver operating characteristic

CRP C-reactive protein 
WBC White blood cell

RBC Red blood cell

HGB Hemoglobin

MCV Mean corpuscular volume

$\mathrm{MCH}$ Mean corpuscular hemoglobin

MCHC Mean corpuscular hemoglobin concentration

NLR Neutrophil to lymphocyte ratio

\section{Declarations}

\section{Ethics approval and consent to participate}

Ethical approval for the study was obtained from the ethical review committee for the first affiliated hospital of Xinjiang medical university, without the need for specific consent from patients.

\section{Consent for publication}

We declare our consent to publication.

\section{Availability of data and material}

Our data and images support the usability of our articles.

\section{Competing interest}

The authors declare that there is no conflict of interest regarding the publication of this paper.

\section{Funding}

The financial support provided by the Xinjiang Uygur Autonomous Region Natural Science Foundation of China (Grant no. 2017D01C300).

\section{Authors' contributions}

Study concept and design: Hui Guo and Feng Gao. Performed research: Li Gou, Hui Guo and Siqin Lan. Analyzed data: Li Gou and Siqin Lan. Wrote the paper: Li Gou and Hui Guo. All authors read and approved the final manuscript.

\section{Acknowledgments}


The authors gratefully acknowledge the Xinjiang Uygur Autonomous Region Natural Science Foundation of China (Grant no. 2017D01C300).

\section{References}

1. Al Dahouk S, Neubauer H, Hensel A, et al. Changing epidemiology of human brucellosis, Germany, 1962-2005. Emerg Infect Dis 2007;13:1895-900.

2. Deqiu S, Donglou X, Jiming Y. Epidemiology and control of brucellosis in China. Vet Microbiol 2002; 90:165-82.

3. Wang Y, Zhang W, Ke Y, et al. Human brucellosis, a heterogeneously distributed, delayed, and misdiagnosed disease in China. Clin Infect Dis 2013; 56:750-1.

4. Yilmaz O, Mehmet C, Kelekci S. Association between red blood cell distribution width and polycystic ovary syndrome. Endocr Res 2014; 22:1-7.

5. Peng YF, Zhang Q, Cao L,et al. Red blood cell distribution width: a potential marker estimating disease activity of ankylosing spondylitis. Int J Clin Exp Med 2014;7:5289-95.

6. Sahin O, Akpek M, Sarli B, et al. Association of Red Blood Cell Distribution Width Levels with Severity of Coronary Artery Disease in Patients with Non-ST Elevation Myocardial Infarction. Med Princ Pract 2014;12:16.

7. Peng YF, Zhang ZX, Cao W, Meng CR, Xu SS, Zhang Q. The association between red blood cell distribution width and acute pancreatitis associated lung injury in patients with acute pancreatitis. Open Med 2015;10:176-179.

8. Gerede DM, Kaya CT, Vurgun VK,et al. Red cell Distribution Width as a Predictor of Left Atrial Spontaneous Echo Contrast in Echocardiography. Medicine (Baltimore) 2015; 94:e712.

9. Galińska EM, Zagórski J. Brucellosis in humans-etiology, diagnostics, clinical forms. Ann Agric Environ Med 2013;20:233-238.

10. Olt S, Ergenç H, AçJkgöz SB. Predictive Contribution of Neutrophil/Lymphocyte Ratio in Diagnosis of Brucellosis. BioMed Res Int 2015;2015:210502.

11. Mohsenpour B, Hajibagheri K, Afrasiabian S, Ghaderi E, Ghasembegloo S. ABO Blood Groups and Susceptibility to Brucellosis.Jpn J Infect Dis 2015;68:124-7.

12. Aktar F, Tekin R, Bektaş MS, et al. Diagnostic role of inflammatory markers in pediatric Brucella arthritis. Ital J Pediatr 2016; 42: 3.

13. Küçükbayrak $A$, Taş $T$, Tosun $M$, et al. Could thrombocyte parameters be an inflammatory marker in the brucellosis? Med Glas (Zenica) 2013,10:35-39.

14. Okan DH, Gökmen Z, Seyit B, Yuksel K, Cevdet Z, Deniz A. Mean platelet volume in brucellosis: correlation between brucella standard serum agglutination test results, platelet count, and $\mathrm{C}$-reactive protein.Afr Health Sci 2014;14: 797-801.

15. Togan T, Narci H, Turan H, Ciftci O, Kursun E, Arslan H.The Impact of Acute Brucellosis on Mean Platelet Volume and Red Blood Cell Distribution. Jundishapur J Microbiol 2015;20:e20039. 
16. Küçükbayrak A, Taş T, Tosun M, Aktaş G, Hakyemez iN, Mengeloğlu FZ. Erythrocytes Parameters in The Course of Brucellosis. Abant Med J 2013;2:36-9.

17. Malandrino N, Wu WC, Taveira TH, Whitlatch HB, Smith RJ. Association between red blood cell distribution width and macrovascular and microvascular complications in diabetes. Diabetologia 2012; 55:226-35.

18. Narci H, Turk E, Karagulle E, Togan T, Karabulut K.The role of red cell distribution width in the diagnosis of acute appendicitis: a retrospective case-controlled study.World Journal of Emergency Surgery 2013; 8:46.

19. Öztürk ZA, Ünal A, Yiğiter R, et al. Is increased red cell distribution width (RDW) indicating the inflammation in Alzheimer's disease (AD)? Arch Gerontol Geriatr 2013; 56:50-4.

20. Harmanci O, Kav T, Sivri B. Red cell distribution width can predict intestinal atrophy in selected patients with celiac disease. J Clin Lab Anal 2012; 26:497-502.

21. 21. Kim J, Kim K, Lee JH, et al. Red blood cell distribution width as an independent predictor of allcause mortality in out of hospital cardiac arrest. Resuscitation 2012; 83:1248-52.

22. Tekin R, Aktar F, Ayaz C. Comparison of Inflammatory Markers Between Adult and Pediatric Brucellosis Patients.Open Forum Infect Dis 2017;4:S350-1.

23. Cift A, Yucel MO. Comparison of inflammatory markers between brucella and non-brucella epididymo-orchitis. Int Braz J Urol 2018; 44: 771-8.

24. Patel KV, Ferrucci L, Ershler WB, Longo DL, Guralnik JM. Red blood cell distribution width and the risk of death in middle-aged and older adults. Arch Intern Med 2009; 169:515-23.

25. Sadaka F, O'Brien J, Prakash S. Red cell distribution width and outcome in patients with septic shock. J Intensive Care Med 2013;28:307-13.

26. Perlstein TS, Weuve J, Pfeffer MA, Beckman JA. Red blood cell distribution width and mortality risk in a community-based prospective cohort. Arch Intern Med 2009; 169:588-94.

27. Lippi G, Targher G, Montagnana M, Salvagno GL, Zoppini G, Guidi GC. Relation between red blood cell distribution width and inflammatory biomarkers in a large cohort of unselected outpatients. Arch Pathol Lab Med 2009; 133:628-32.

\section{Figures}




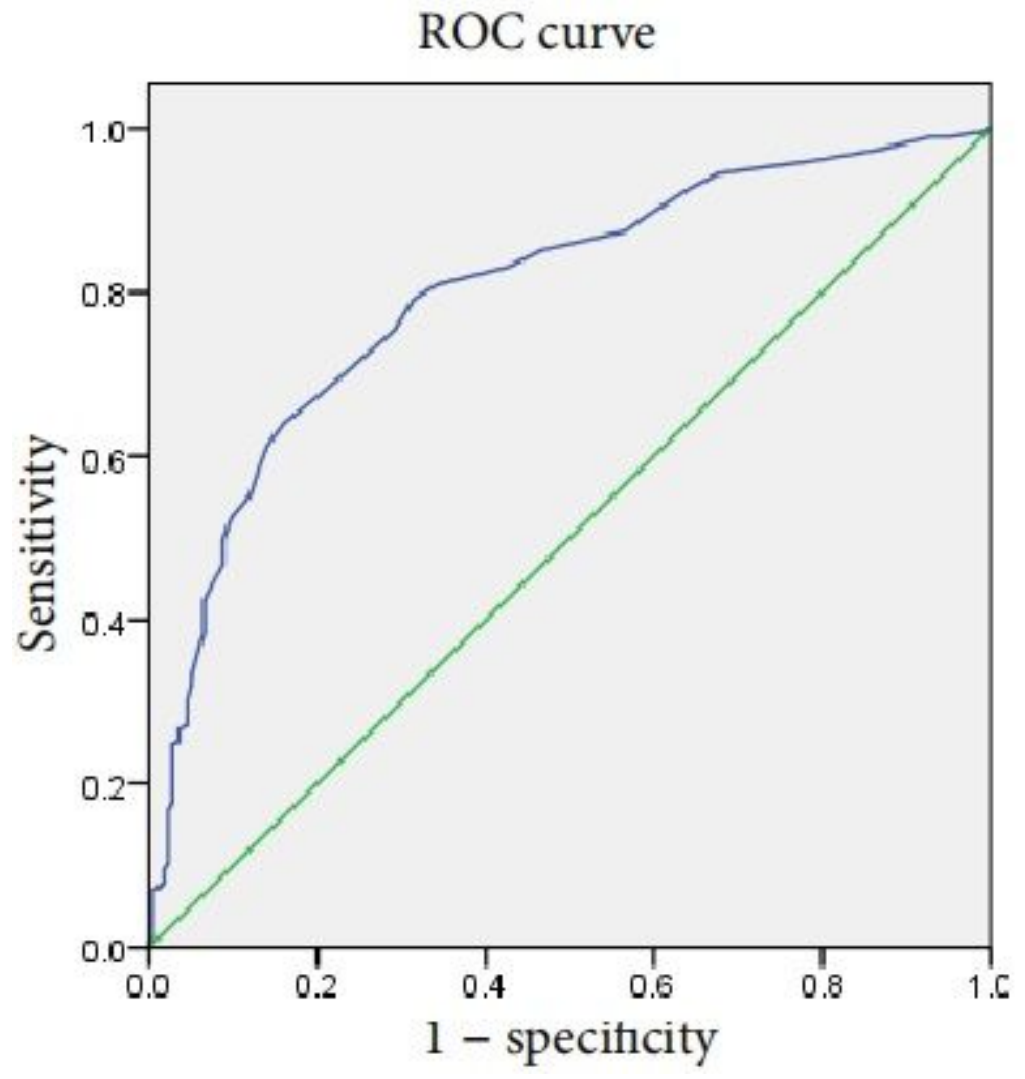

Figure 1

Receiver operating characteristic curve analysis of red blood cell distribution width. 